\title{
Upcoming Meetings
}

Aorta

AORTA, June 2016, Volume 4, Issue 3:114

DOI: http://dx.doi.org/10.12945/j.aorta.2016.16.028

Received: June 1, 2016

Accepted: June 1, 2016

Published online: June, 2016

\section{List of Upcoming Meetings}

\section{November 2016}

1. EACTS Academy: Aortic Valve Surgery

November 24-25, 2016

Nancy, France

Meeting information available at:

www.eacts.org/academy/courses/aortic-

valve-surgery

\section{December 2016}

1. International Conference for Innovations in Cardiovascular Systems

December 4-6, 2016

Tel Aviv, Israel

Meeting information available at:

2016.icimeeting.com

2. 13th European Cardiology Congress

December 5-6, 2016

Madrid, Spain

Meeting information available at:

cardiology.conferenceseries.com/europe

\section{January 2017}

1. 35th Annual International Symposium: Clinical Update in Anesthesiology, Surgery and Perioperative Medicine

January 15-20, 2017

Cancun, Mexico

Meeting information available at:

www.clinicalupdateinanesthesiology.org

2. Controversies and Updates in Vascular Surgery January 19-21, 2017

Paris, France

Meeting information available at:

cacvs.org

3. 53rd Annual Meeting of the Society of

Thoracic Surgeons and STS/AATS

Tech-Con 2017

January 21-25, 2017

Houston, Texas

Meeting information available at:

www.sts.org/education-meetings/sts-annual-

meeting

4. STS/CTSNet Career Fair at the 53rd

Annual Meeting

January 22-24, 2017

Houston, Texas

Meeting information available at:

www.ctsnet.org/events/2017-sts-and-ctsnet-

career-fair 